\title{
SIMPLE PROOF AND REFINEMENT OF HERMITE-HADAMARD INEQUALITY
}

\author{
ABDALLAH EL FARISSI
}

Abstract. In this note we give a simple proof and a new generalization of the Hermite-Hadamard inequality.

Mathematics subject classification (2010): 52A40, 52A41.

Keywords and phrases: Convex functions, Hermite-Hadamard integral inequality.

\section{REFERENCES}

[1] M. Bessenyei And Zs. PÁLes, Higher-order generalizations of Hadamard's inequality, Publ. Math. Debrecen, 61, 3-4 (2002), 623-643.

[2] M. BessenYeI AND Zs. PÁLES, Hadamard-type inequalities for generalized convex functions, Math. Inequal. Appl., 6, 3 (2003), 379-392.

[3] S. S. Dragomir and C. E. M. Pearce, Selected Topics on Hermite-Hadamard Inequalities, (RGMIA Monographs http://rgmia.vu.edu.au /monographs/hermite_hadamard.html), Victoria University, 2000.

[4] A. El Farissi, Z. Latreuch, B. Belaidi, Hadamard-Type Inequalities for Twice Differentiable Functions, RGMIA Research Report collection, 12, 1 (2009), Art. 6.

[5] A. M. FINK, A best possible Hadamard inequality, Math. Inequal. Appl., 1, 2 (1998), 223-230.

[6] J. HADAMARD, Étude sur les propriétés des fonctions entières et en particulier d'une fonction considérée par Riemann, J. Math. Pures Appl., 58 (1893), 171-215.

[7] D. S. Mitrinović And I. B. Lacković, Hermite and convexity, Aequationes Math., 28 (1985), 229-232.

[8] C. Niculescu And L.-E. Persson, Old and new on the Hermite-Hadamard inequality, Real Analysis Exchange, 2004.

[9] C. Niculescu And L.-E. Persson, Convex Functions and Their Applications. A Contemporary Approach, CMS Books in Mathematics, Vol. 23, Springer-Verlag, New York, 2006.

[10] J. E. PeČarić, F. Proschan and Y. C. Tong, Convex Functions, Partial Orderings and Statistical Applications, Academic Press, New York, 1992.

[11] J. ŚANDOR, Some integral inequalities, El. Math., 43 (1988), 177-180. 